Received: 2017.11 .12

Accepted: 2018.01.03

Published: 2018.07.26

\title{
Astragalus Polysaccharide Protects Neurons and Stabilizes Mitochondrial in a Mouse Model of Parkinson Disease
}

Authors' Contribution: Study Design A Data Collection B Statistical Analysis C Data Interpretation D Manuscript Preparation E Literature Search F Funds Collection $G$

\author{
ABCE 1,2,3 Hong Liu \\ BCD 4 Si Chen \\ CD 2,3 Cunju Guo \\ $B C D 5$ Wenqiang Tang \\ EF 5 Wei Liu \\ ABCDEFG 1 Yiming Liu
}

\author{
1 Department of Neurology, Qilu Hospital of Shandong University, Jinan, Shandong, \\ P.R. China \\ 2 Department of Neurology, People's Hospital of Liaocheng Affiliated to Taishan \\ Medical College, Liaocheng, Shandong, P.R. China \\ 3 Department of Neurology, First Hospital of Liaocheng University, Liaocheng, \\ Shandong, P.R. China \\ 4 Department of Neurosurgery, Qilu Hospital of Shandong University, Jinan, \\ Shandong, P.R. China \\ 5 Department of Central Laboratory, People's Hospital of Liaocheng Affiliated to \\ Taishan Medical College, Liaocheng, Shandong, P.R. China
}

Corresponding Author: Source of support:

Background: Astragalus polysaccharides (APS) have a very good therapeutic effect in the treatment of neurodegenerative diseases and nerve injury disease. However, research on Parkinson disease (PD) treatment with APS is lacking.

Material/Methods: The present study was designed to explore the effects of APS on the protection of neurons and mitochondrial in a mouse model of PD using behavioral experiments, and observations of mitochondrial structure and transmembrane potential.

Results: It was shown that APS could attenuate 1-methyl-4-pheyl-1,2,3,6-tetrahydropyridine (MPTP)-induced motor dysfunction $(P<0.01)$, increase the proportion of TH-positive cells $(P<0.01)$, reverse MPTP-induced mitochondrial structural damage, and reduce MPTP-induced high levels of reactive oxygen species (ROS) and increase MPTPinduced decrease in mitochondrial membrane potential. In addition, APS also decreased the bax/bcl2 ratio, and cytochrome-c and caspase-3 protein content $(P<0.01)$ in substantia nigra in our mouse PD model.

Conclusions: $\quad$ APS provided a protective effect on neurons and mitochondrial in a mouse PD model.

MeSH Keywords: Mitochondrial Diseases • Parkinson Disease • Polysaccharides

Full-text PDF: https://www.medscimonit.com/abstract/index/idArt/908021

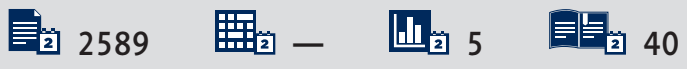




\section{Background}

Parkinson disease (PD) is a central nervous system degenerative disease that mainly occurs in middle-aged and elderly people. It effects multiple neurons in the early stage, with central nervous system damage as the main clinical manifestation in the late or advanced stage [1,2]. The formation of residual markers of dopaminergic neurons in the cytoplasm and the progressive degeneration of dopaminergic neurons in the densities of midbrain substantia nigra are characterized by PD typical pathologic changes [1,2]. Although the pathogenesis of PD is not clear, many studies [3-5] have shown that mitochondrial damage plays an important role in the pathogenesis of PD. Mitochondrial dysfunction and oxygenated stress damage are the main pathological mechanisms of nervous system degenerative diseases. Mitochondria, as organelles that are abundantly distributed in neuronal cells, have long been considered "energy metabolism plants" that supports nerve cell function through oxidative phosphorylation [6,7]. In addition to participating in energy metabolism in cells, it also plays an important physiological and pathological mediating role in the regulation of cell calcium homeostasis [8], free radical dynamic balance [9], apoptosis [10], etc. [11].

Recently, more and more scholars have begun to study the mechanism of traditional Chinese medicine and its monomer treatment of PD. These mechanisms are mainly involved in protecting dopaminergic neurons [12], improving mitochondrial function [13], reducing neuritis [14], enhancing immune response [15], reducing excitotoxicity [16], anti-apoptosis [17], inducing autophagy [18], and inhibiting the accumulation of abnormal proteins [19]. For example, geniposide enhances the growth factor signal and reduces apoptosis in a neuroprotective effect. Paeoniflorin may protect neurons by enhancing mitochondrial function.

Astragalus polysaccharide (APS) is one of the main active ingredients in astragalus. It has been reported to have many pharmacological activities [20-23], such as immunity enhancement, anti-tumor, hypoglycemic, anti-oxidation, anti-inflammatory, anti-aging, and antiviral activities, and has a wide range of clinical applications. At present, the research and clinical application of APS has mostly focused on the regulation of immune function. The treatments available for nerve-related diseases are few, and clinical development and application is lacking. However, APS has been reported to have good prevention and improvement effects on ischemic stroke, Alzheimer disease, and some nerve injury symptoms [24,25].

Based on these reports, the present study was performed to evaluate the effects of APS on the protection of neurons and mitochondrial function in a mouse PD model.

\section{Material and Methods}

\section{Animal model of PD and drug administration}

C57B/6 mice (male, 5-8 weeks old) were used to make the animal models for this study; they were fed and housed at $22 \pm 2^{\circ} \mathrm{C}$, with a12-hour light cycle at an animal experimental center. All animal protocols were approved by the Animal Care and Use Committee in the hospital and conformed with the guidelines of the National Institution of Health.

The mice were divided into 4 groups. Mice were injected with 1-methyl-4-pheyl-1,2,3,6-tetrahydropyridine (MPTP) intraperitoneally ( $25 \mathrm{mg} / \mathrm{kg}, 1$ time/day for 7 days) to make the PD model. The mice in the control group were given the same dose of physiological saline in the first 5 days. On days 8 to 21 of the experiment, APS (10 mg/kg, 1 time/day) was intraperitoneally injected into the mice in the MPTP+APS group and the control+APS group, and the same dose of physiological saline was injected into the control group and the MPTP group.

\section{Behavioral experiment}

\section{Climb pole testing}

The climb pole test was used to detect mouse movement coordination ability 1 day after dosing; the mice were adapted to the test environment 2 days before a test. A piece of wood $55 \mathrm{~cm}$ long and $1 \mathrm{~cm}$ in diameter was used for testing, and a wooden ball with a diameter of $2.5 \mathrm{~cm}$ was fixed at the top of the wood; cotton yarn was wrapped around the wood to prevent the mice from slipping. At the time of the test, we recorded the time it took for the mouse to climb from the bottom of the ball to the bottom of the wood as $T_{L A}$. The test was repeated 3 times, with a 5 -minute rest between each time.

\section{Open field testing}

The open field test was used to detect the physical activity of the mice and the degree of mental depression. An uncapped square box with a size of $40 \times 40 \times 10 \mathrm{~cm}$ was prepared for the testing, and the bottom of the box was divided into 16 parts. At the beginning of the test, the mouse was placed in the left rear area of the box, and then allowed free exercise for $5 \mathrm{~min}$ utes. The mouse speed $(\mathrm{V})$ and the residence time in the central region $(\mathrm{T})$ were recorded.

\section{TH-positive neurons in substantia nigra}

On the second day after the behavioral testing, the mice were anesthetized by intraperitoneal injection of $1 \%$ pentobarbital sodium (10 mg/kg), the chest was quickly opened, and a saline rinse applied. A total of $40 \mathrm{~mL}$ of $4 \%$ paraformaldehyde 
phosphate buffer fixative was used to infuse the mice after the liver became white. After the limbs were stiff, we quickly removed the brain tissue, and fixed it in $4 \%$ paraformaldehyde phosphate buffer for 48 hours, and $20 \%$ sucrose solution overnight in $4^{\circ} \mathrm{C}$. Then tissue slices were made using a thermostat slicer (SLEE, CUT4062, Germany).

The slices were warmed in $0.01 \mathrm{M}$ PBS for 0.5 hours and rinsed 3 times with PBS (10 minutes each time) before immunocytochemistry. The substantia nigra slices were stained with rabbit anti-TH polyclonal antibody (ABCAM, UK) which was diluted by $3 \%$ BSA and $0.3 \%$ Triton X-100 (1: 400), followed by fluorescein labeled donkey anti-rabbit IgG antibody (1: 500, Abcam, Ltd., UK). The fluorescence microscopy (BX51, OLYMPUS, Japan) was used to observe the staining results, and $\mathrm{TH}$-positive neurons were counted by Photoshop CS 5 (Adobe, Ltd., USA).

\section{Mitochondrial structure}

The substantia nigra was obtained as previously described, then placed in $2.5 \%$ glutaraldehyde for 24 hours in $4^{\circ} \mathrm{C}$, followed by $2 \%$ osmic acid for 2 hours at room temperature, $30 \%$ and $50 \%$ ethanol dehydration for 15 minutes, $70 \%$ uranyl acetate saturated with ethanol for 12 hours, $80 \%$ and $95 \%$ ethanol dehydration for 15 minutes, $100 \%$ ethanol dehydration 2 times, once for 40 minutes, epichlorohydrin for 30 minutes, and then embedded with Epon 812 epoxy embedding kit. The slices were obtained using an ultra-thin slicer (LKB-III, LKB, Sweden), and were double-stained with uranium acetate and lead nitrate. The transmission electron microscopy $(\mathrm{H}-7500$, Hitachi, Ltd., Japan) was used to observe the mitochondrial structure.

\section{Mitochondrial transmembrane potential}

The substantia nigra was obtained as previously described. We added $0.25 \%$ trypsin (HyClone, USA) for 30 minutes in $37^{\circ} \mathrm{C}$, terminated the incubation with cell culture medium, cells filtered using 200 mesh cell sieves, and cells collected by centrifugation at 1000 rpm, for 5 minutes (Eppendorf, Germany). A total of $1 \times 10^{6}$ cells was diluted by 100 uL PBS buffer for mitochondrial membrane potential detection, then $10 \mathrm{ug} / \mathrm{mL}$ Rho123 buffer (SIGMA, Germany) was added to cells for 30 minutes, incubated at $37^{\circ} \mathrm{C}$, and the mitochondrial membrane potential was detected by flow cytometry (Miltenyi Biotec, Germany). The average fluorescence intensity was used to represent the mitochondrial membrane potential.

\section{Reactive oxygen species (ROS)}

The substantia nigra was obtained as previously described. The reactive oxygen species (ROS) of mitochondrial in neurons of substantia nigra was detected by ROS Assay Kit (Sigma, Germany).

\section{Western blot}

The substantia nigra was placed in a $2 \mathrm{~mL}$ tube. Total Protein Extraction Kit-tissue (Beyotime, China) was used to extract the total protein. The concentration of total protein was detected by BCA Protein Assay Kit (Beyotime, China). Protein were analyzed using SDS-PAGE (300 mA, 1.5 hours) and western blotting; with bax, bcl-2, cytochrome-c, and caspase- 3 rabbit monoclonal antibodies (ABCAM, UK) as the first antibody, and alkaline enzyme labeled horse anti-rabbit IgG (ABCAM, UK) as the second antibody.

\section{Drugs and data analysis}

MPTP, pentobarbital sodium, sucrose, Triton X-100, osmic acid, and all other chemicals and reagents were purchased from Sigma-Aldrich (Sigma, Germany). APS was isolated and purified as described previously [20].

All data were analyzed by SPSS20.0; $t$-test or one-way analysis of variance ANOVA was used to compare differences between groups for the data on behavioral scoring, TH-positive neuronal ratio, mitochondrial ROS, mitochondrial membrane potential, and protein expression levels. A $P$ value less than 0.05 was considered a significant difference.

\section{Results}

\section{APS attenuated MPTP-induced motor dysfunction}

The mice were observed to have gradual exercise reduction, limb tremor, bow and gait instability, and other motor dysfunction symptoms after intraperitoneally treated with MPTP, and the symptoms gradually improved after APS treatment.

As shown in Figure 1, the $T_{L A}$ of the mice in the MPTP group was $33.67 \pm 3.81$ seconds, which was significantly higher than in the control group $(15.33 \pm 1.68$ seconds $P<0.01)$. After treatment with APS for 14 days, the $T_{L A}$ of the mice in the MPTP+APS group decreased to $24.67 \pm 2.85$ seconds (Figure $1 \mathrm{~A}$ ). The $T$ of the mice in the MPTP group was $13.93 \pm 1.91$ seconds, which was significantly higher than in the control group $(5.06 \pm 1.22$ seconds, $P<0.01)$. After treatment with APS for 14 days, the T of the mice in the MPTP+APS group was decreased to $10.4 \pm 1.88$ seconds (Figure1B). The $V$ of the mice in the MPTP group was $1.99 \pm 0.47$ seconds, which was significantly less than in the control group $(5.49 \pm 0.55$ seconds, $P<0.01)$. After treatment with APS for 14 days, the $V$ of the mice in the MPTP+APS group increased to $4.48 \pm 0.49$ seconds (Figure $1 C$ ). 


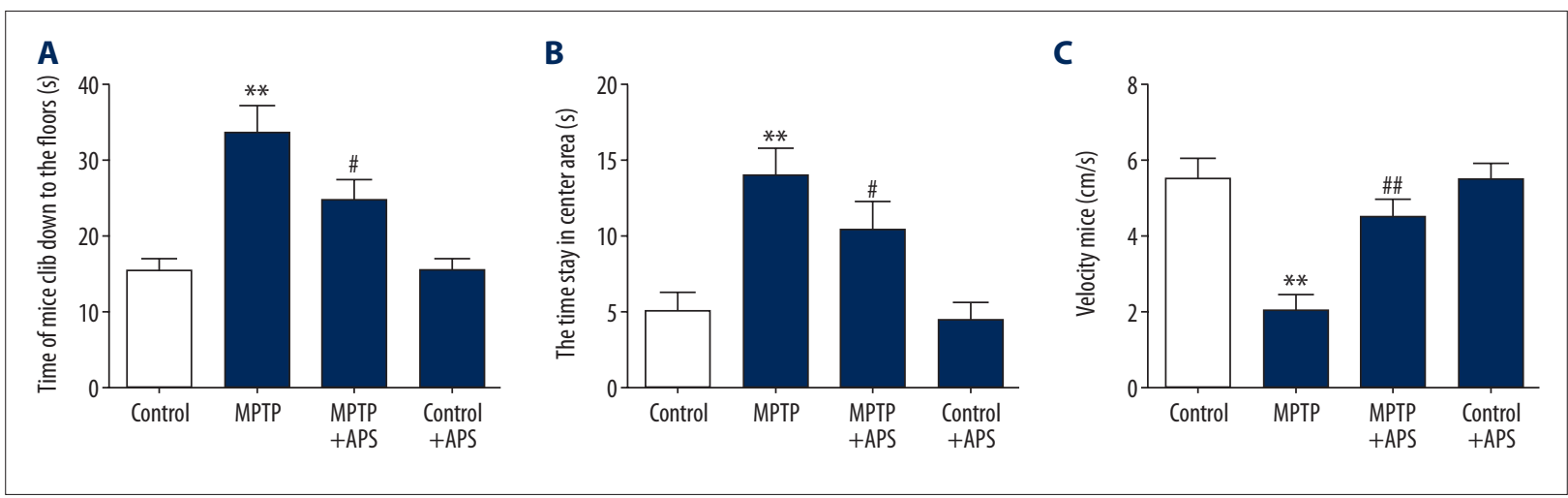

Figure 1. Effects of APS on MPTP-induced behavioral injury. The time it takes mice to climb down to the flow in climb pole testing (A). The time mice stay in the center area of the uncapped square box in open field testing (B). The speed of mice in the uncapped square box in open field testing (C). ${ }^{* *} P<0.01$ compared to the control group, ${ }^{\#} P<0.05$ and ${ }^{\# \#} P<0.01$ compared to the MPTP group.

\section{APS attenuated MPTP-induced TH-positive neurons decreased}

TH-positive neurons were mainly distributed in SNpc, which was observed using fluorescent fiber. After intraperitoneal treatment with MPTP, the TH-positive neurons of substantia nigra were decreased to $36.67 \%$ compared to the control group. However, TH-positive neurons of substantia nigra were increased to $64.31 \%$ compared to the control group after APS treatment (Figure 2).

\section{APS attenuated MPTP-induced neurons mitochondrial structure change}

Mitochondrial structure changes were macroscopic manifestations of mitochondrial damage. In the control group, the neuron mitochondrial structure in substantia nigra were small, round or oval, with structural integrity and uniform density of the matrix electrons, following ridge distribution rules. After treatment with MPTP, the mitochondrial chamber expanded, the electron density was significantly reduced, and the mitochondria structure changed to loose vacuoles, and there was obvious swelling; mitochondrial crista became short and less. Treatment with APS could reverse these changes in the mitochondrial structure. First, mitochondrial edema was significantly reduced when compared with the MPTP group. Second, the mitochondrial structure was basically complete in the MPTP+APS group, and only part of the mitochondrial crest became shorter or fractured. Third, the mitochondrial matrix density was still reduced (Figure 3).

\section{Effects of APS on mitochondrial function of neurons}

After intraperitoneal treatment with MPTP, the ROS level was increased by $51.87 \%$ vs. the control group, and the mitochondrial transmembrane potential was decreased by 15.53 vs. the control group. After APS treatment, the ROS level decreased by $20.63 \%$ vs. the MPTP group, and the mitochondrial transmembrane potential increased by 8.27 vs. the MPTP group (Figure.4).

\section{Effects of APS on changes of bax/bcl-2 and expression of cytochrome and caspase- 3 in the MPTP-induced mice}

As show in Figure 5, the expression of bax, cytochrome-c, procaspase-3, and caspase- 3 protein increased significantly after induction by MPTP, but the expression of bcl- 2 was just the opposite. Seven days after injection of MPTP, the mice were injected with APS for 2 consecutive weeks, then we found the expression of bax, cytochrome-c, pro-caspase-3, and caspase- 3 protein decreased significantly, compared with the control group. $\mathrm{Bcl}-2$ increased significantly.

\section{Discussion}

PD is a neurodegenerative disease that mainly occurs in middle-aged and elderly patients and is characterized by a loss of projecting dopaminergic neurons in the substantia nigra. Mitochondrial dysfunction is one of the main mechanisms of PD. Protecting and repairing damaged neurons and mitochondria is the primary pathway for the prevention and treatment of PD [1-5].

APS is extracted from astragalus, and its injection has a wide range of clinical applications. APS not only increases the growth and number of neuronal axons by promoting the secretion of nerve growth factor [26], but it also may play a vital role in neuronal growth, as well as survival and injury repair by promoting the secretion of glial cell-derived neurotrophic factor [25]. In addition, APS may suppress mitochondrial ROS in mesenchymal stem cells [27], inhabit liver mitochondrial permeability transition, and scavenge superoxide anion, hydroxyl 

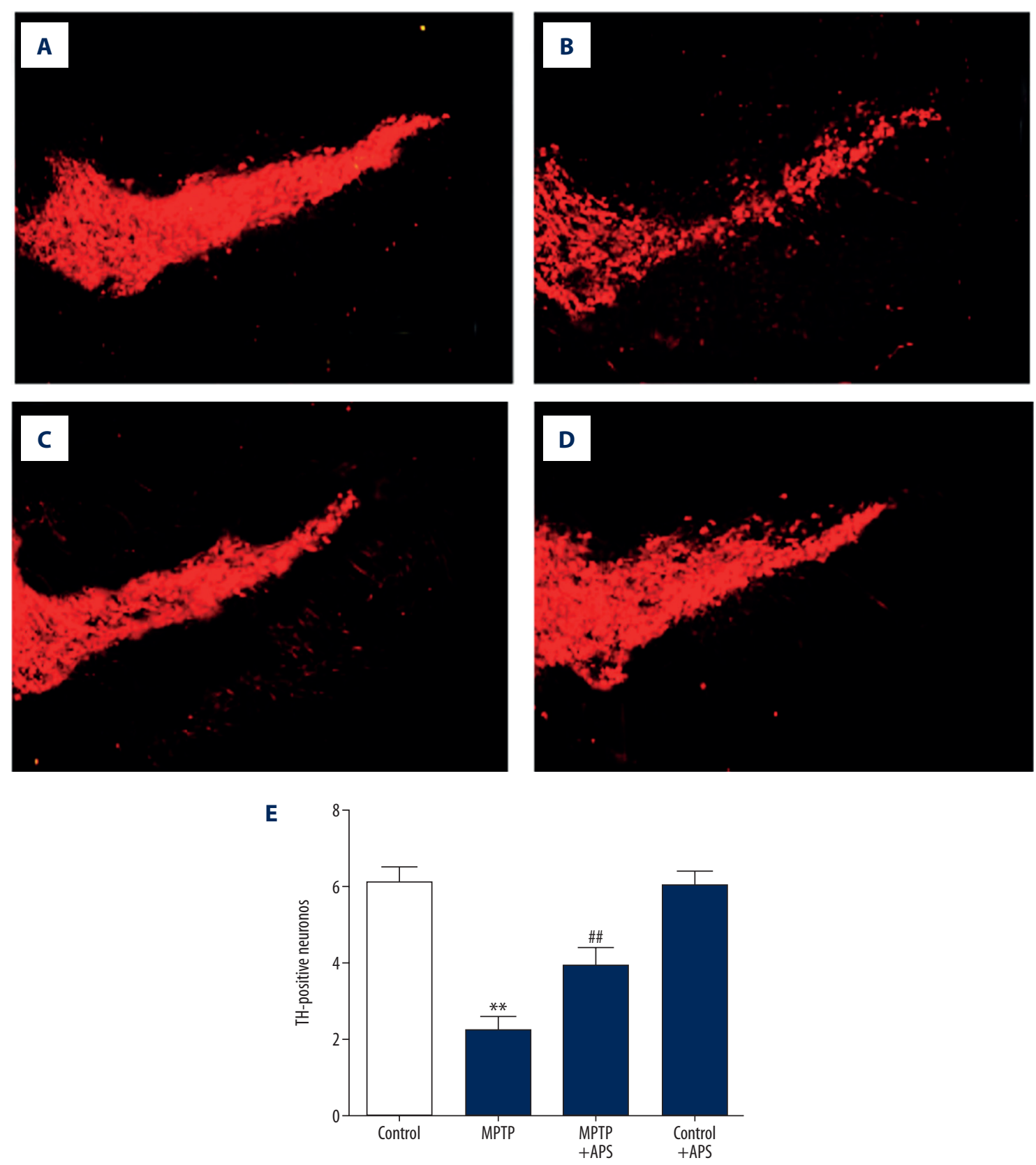

Figure 2. APS attenuated MPTP-induced TH-positive neurons of substantia nigra were decreased. TH-positive neurons in the substantia nigra distributed in the control group (A), MPTP group (B), MPTP+APS group (C), and control+APS group (D). Quantification of TH-positive neurons in the substantia nigra for each group (E). ${ }^{* *} P<0.01$ compared to the control group, and \#\# $P<0.01$ compared to the MPTP group. Bar: $100 \mu \mathrm{m}$.

radicals, and hydrogen peroxide [28]. Thus, APS has potential value in the treatment of PD.

The mouse model of PD in this study was prepared by intraperitoneal injection of MPTP. MPTP is a fat-soluble neurotoxic agent, which can quickly penetrate the blood-brain barrier and produce a toxic effect of MPP+ by metabolism. Dopaminergic neurons in the substantia nigra would then undergo abnormal change or absence because of reduced ATP and risen ROS when MPP+ enters dopaminergic neurons and is absorbed and concentrated by the mitochondria. But we found that APS not only attenuated MPTP-induced motor dysfunction, but also attenuated MPTP-induced tyrosine hydrogenase (TH)-positive neurons decrease. PD would appear when $50 \%$ of dopaminergic 

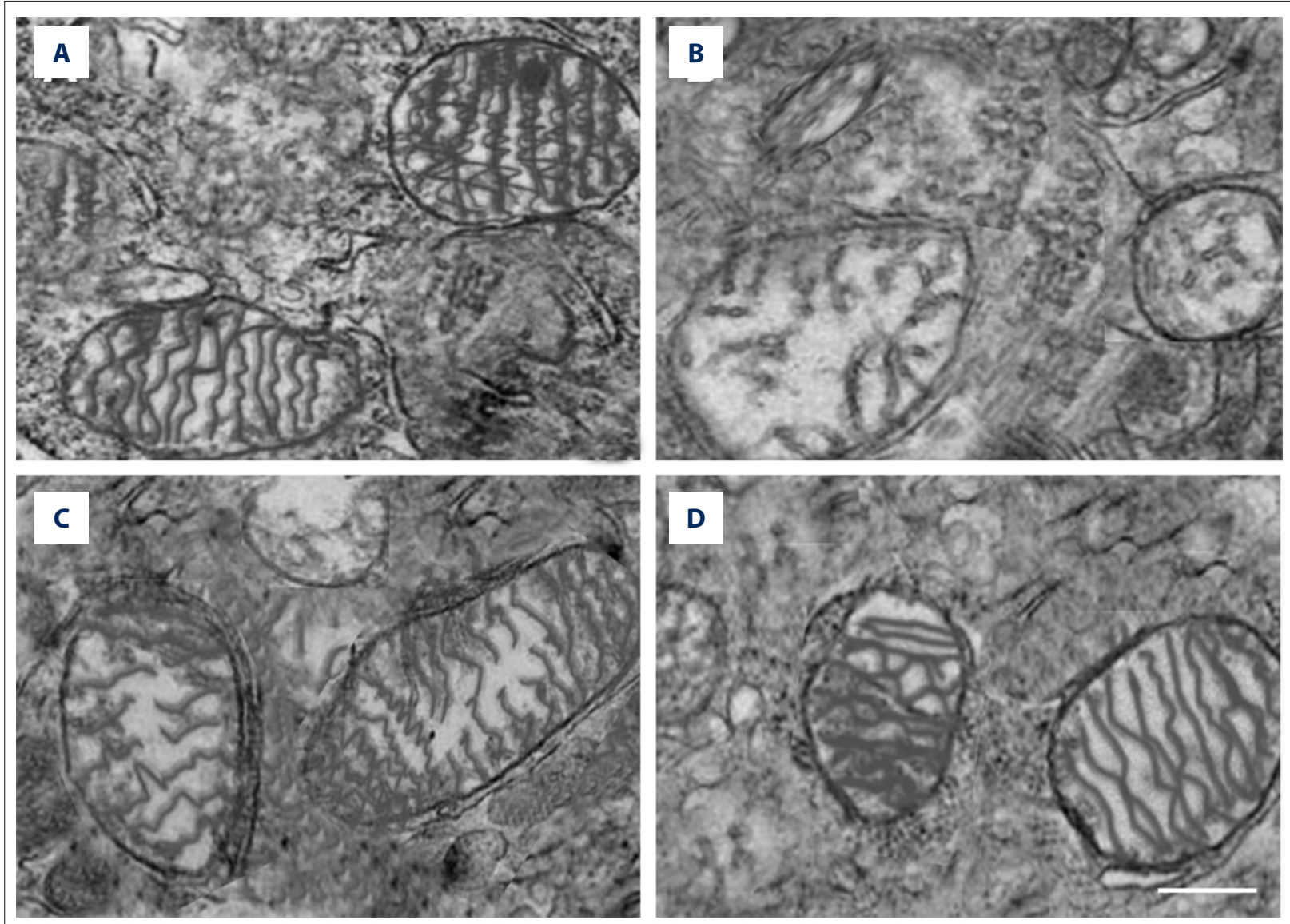

Figure 3. APS reversed the changes of mitochondrial structure induce by MPTP. The neurons mitochondrial structure of substantia nigra in the control group (A), MPTP group (B), MPTP+APS group (C), and control+APS group (D) by transmission electron microscopy. Bar: $1 \mu \mathrm{m}$.

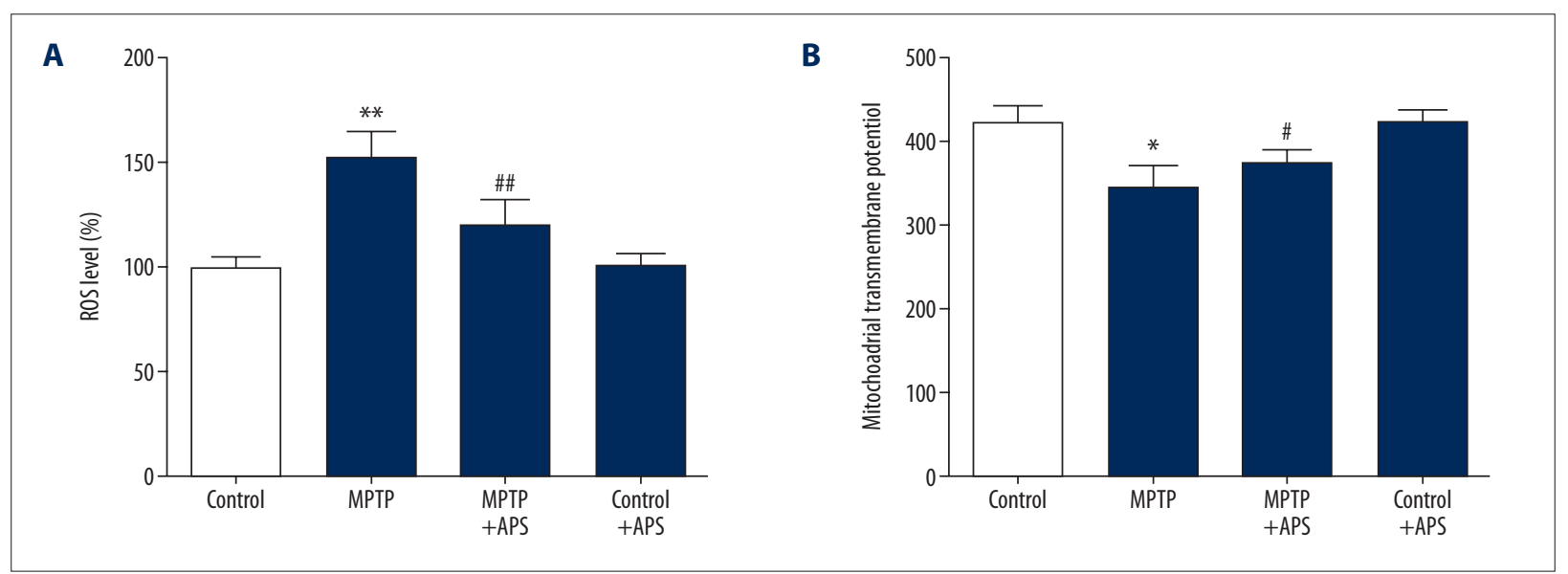

Figure 4. APS repaired MPTP-induced mitochondrial dysfunction. The ROS levels are expressed as the percent of the control group (A). The mitochondrial transmembrane potential was detected by flow cytometry and represented as the average fluorescence intensity (B). ${ }^{* *} P<0.01$ and ${ }^{*} P<0.05$ compared to the control group, ${ }^{\#} P<0.05$ and ${ }^{\# \#} P<0.01$ compared to the MPTP group. 


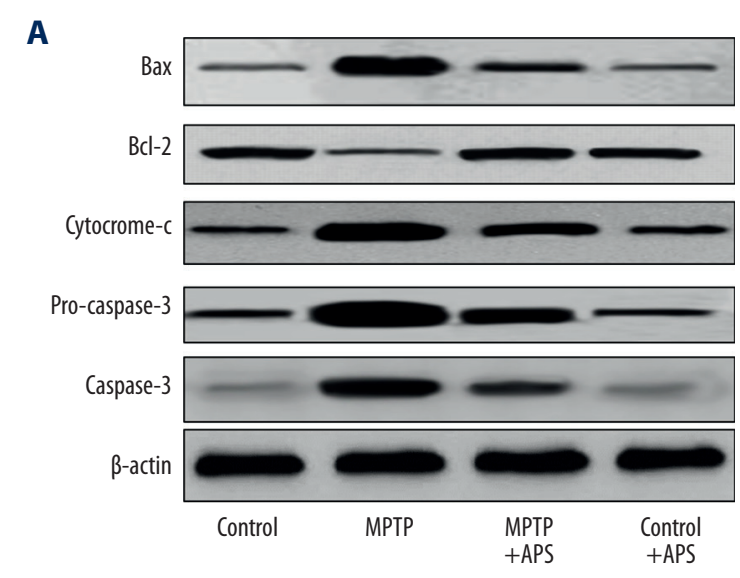

B

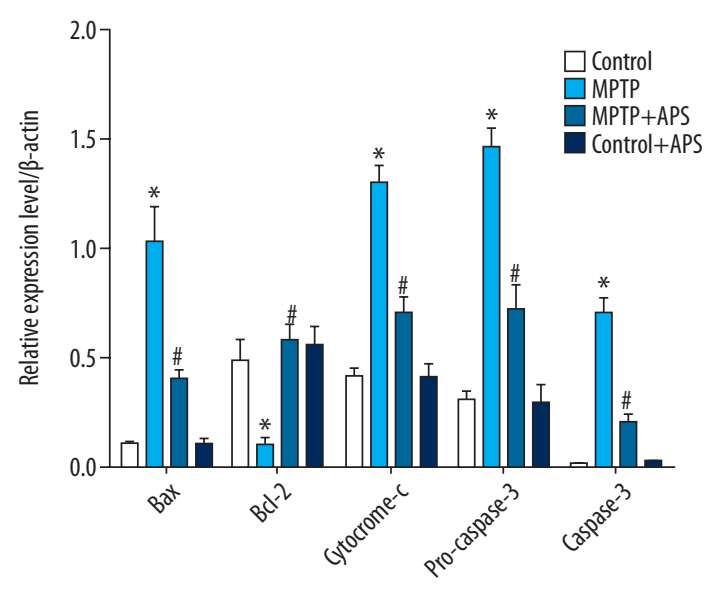

Figure 5. APS reversed the changes of protein express induce by MPTP: western blot results (A); the statistical analysis of the western blot results (B). * $P<0.05$ compared to the control group, ${ }^{\#} P<0.05$ compared to the MPTP group.

neurons were missing in substantia nigra [29]. And TH was a synthetic rate limiting enzyme of dopaminergic neurons, which was positively correlated with dopaminergic neurons. In the present study, TH-positive cells in the PD model that had motor dysfunction accounted for only $36.67 \%$ of health mice. Mice treated with APS not only improved in motor dysfunction, but also $\mathrm{TH}$-positive cells increased by $75.44 \%$. This suggests that there was a closely relation between motor dysfunction and the absence of TH-positive cells in our mouse model of PD. Thus, promoting the proliferation of $\mathrm{TH}$-positive cells would help in the prevention and treatment of PD [30-32].

Mitochondria is an important organelle for oxidative phosphorylation and energy production of cells. It plays an important role in maintaining normal physiological function of cells. Mitochondrial plays a normal function only with a complete structure; however, when a series of dysfunctions occurs, the mitochondrial structure is destroyed $[33,34]$. In the present study, we found that the mitochondrial structure in neurons was destroyed, mitochondrial membrane potential was decreased, and mitochondrial ROS was increased in the MPTPinduced mouse model of PD. OS has been shown to be associated with the pathogenesis of a variety of neurodegenerative diseases [35,36], including PD [37]. ROS, coming from OS, could cause DNA fragmentation, lipid autoxidation and mitochondrial dysfunction, and the lack of mitochondrial function will further produce more ROS [35-37]. In our study, we found that APS attenuated the MPTP-induced raised ROS. Thus, APS can be used to treat PD by suppressing the production of ROS, but the molecular mechanism is unclear.
The normal mitochondrial transmembrane potential (MTP) is based on maintaining the mitochondria for oxidative phosphorylation and ATP production, which is necessary for normal mitochondrial function. The protein of cytochrome-c in the cytoplasm increases because of decreasing MTP, which then activates caspase-3, eventually causing apoptosis [38]; as was shown in our study.

In addition, our study also found that APS could reduce the bax/bcl2 ratio in the substantia nigra in a PD mouse model. $\mathrm{Bcl} 2$ is an anti-apoptotic protein, and bax is a pro-apoptotic protein. They co-control the apoptosis by regulating the release of cytochrome-c protein, and activation of capase- 3 protein and mitochondrial function $[39,40]$. When the bax/bcl2 ratio increases, cytochrome-c is released from the mitochondria. Caspase- 3 is activated by cytochrome-c, and mitochondrial function is lost, eventually causing apoptosis.

\section{Conclusions}

APS provided protective effects to neurons and mitochondria in a mouse model of PD at a dose of $10 \mathrm{mg} / \mathrm{kg}$ for 14 days. To the best of our knowledge, this is the first study of APS in a mouse model of PD, although there are many studies on the treatment of neurodegenerative diseases by APS.

\section{Conflict of interest}

None. 


\section{References:}

1. Kalia LV, Lang AE: Parkinson's disease. Lancet, 2015; 386: 896-912

2. Grayson M: Parkinson's disease. Nature, 2016; 538: S1

3. Pickrell AM, Youle RJ: The roles of PINK1, parkin, and mitochondrial fidelity in Parkinson's disease. Neuron, 2015; 85: 257-73

4. Matheoud D, Sugiura A, Bellemare-Pelletier A et al: Parkinson's diseaserelated proteins PINK1 and Parkin repress mitochondrial antigen presentation. Cell, 2016; 166: 314-27

5. Lu M, Su C, Qiao C et al: Metformin prevents dopaminergic neuron death in MPTP/P-induced mouse model of Parkinson's disease via autophagy and mitochondrial ROS clearance. Int J Neuropsychopharmacol, 2016; 19: pii: pyw047

6. Kadenbach B: Mitochondrial oxidative phosphorylation. Angewandte Chemie, 2013; 748: 658-62

7. Nagy A, Eder K, Selak MA et al: Mitochondrial energy metabolism and apoptosis regulation in glioblastoma. Brain Res, 2015; 1595: 127-42

8. Oxenoid K, Dong Y, Cao C et al: Architecture of the mitochondrial calcium uniporter. Nature, 2016; 533: 269

9. Lagouge M, Larsson NG: The role of mitochondrial DNA mutations and free radicals in disease and ageing. J Intern Med, 2013; 273: 529-43

10. Renault TT, Floros KV, Elkholi R et al: Mitochondrial shape governs BAXinduced membrane permeabilization and apoptosis. Mol Cell, 2015; 57: 69-82

11. Chan DC: Mitochondria: Dynamic organelles in disease, aging, and development. Cell, 2006; 125: 1241-52

12. Zhao X, Zhai S, An MS et al: Neuroprotective effects of protocatechuic aldehyde against neurotoxin-induced cellular and animal models of Parkinson's disease. PLoS One, 2013; 8: e78220

13. Liu H, Yu C, Xu T et al: Synergistic protective effect of paeoniflorin and $\beta$-ecdysterone against rotenone-induced neurotoxicity in PC12 cells. Apoptosis, 2016; 21: 1354-65

14. Zhang J, Ma YZ, Shen XM: Evaluation on the efficacy and safety of Chinese herbal medication Xifeng Dingchan Pill in treating Parkinson's disease: Study protocol of a multicenter, open-label, randomized active-controlled trial. J Integr Med, 2013; 11: 285-90

15. Xue X, Liu H, Qi L et al: Baicalein ameliorated the upregulation of striata glutamatergic transmission in the mice model of Parkinson's disease. Brain Res Bull, 2014; 103: 54-59

16. Sun A, Xu X, Lin J et al: Neuroprotection by Saponins. Phytother Res, 2015 ; 29: $187-200$

17. Chen Y, Zhang Y, Li L et al: Neuroprotective effects of geniposide in the MPTP mouse model of Parkinson's disease. Eur J Pharmacol, 2015; 768: 21-27

18. Jia L, Min C, Xue W et al: Piperine induces autophagy by enhancing protein phosphotase $2 \mathrm{~A}$ activity in a rotenone-induced Parkinson's disease model. Oncotarget, 2016; 7: 60823

19. Kampen JM, Van, Baranowski DB, Shaw CA et al: Panax ginseng is neuroprotective in a novel progressive model of Parkinson's disease. Exp Gerontol, 2014; 50: 95-105

20. Liu Y, Liu F, Yang Y et al: Astragalus polysaccharide ameliorates ionizing radiation-induced oxidative stress in mice. Int J Biol Macromol, 2014; 68: 209-14

21. Xue H, Gan F, Zhang Z et al: Astragalus polysaccharides inhibits PCV2 replication by inhibiting oxidative stress and blocking NF-KB pathway. Int J Biol Macromol, 2015; 81: 22-30
22. Zhang X, Yao K, Ren L et al: Protective effect of Astragalus polysaccharide on endothelial progenitor cells injured by thrombin. Int J Biol Macromol, 2016; 82: 711-18

23. Wu S, Li Y, Chen S et al: Effect of dietary Astragalus polysaccharide supplements on testicular piRNA expression profiles of breeding cocks. Int J Biol Macromol, 2017; 103: 957-64

24. Fei HX, Gao Y, Sun LH et al: [Effect of Astragalus polysaccharides on the hippocampal tissue in Alzheimer's disease mouse.] Chinese Journal of Gerontology, 2015; 16 [in Chinese]

25. Xiang YX, Xiao LY, Zhang J et al: [Recent progress in protective effects and related mechanism of Astragalus polysaccharide against neurological diseases.] Chinese Journal of Hospital Pharmacy, 2016 [in Chinese]

26. Lu MC, Yao CH, Wang SH et al: Effect of Astragalus membranaceus in rats on peripheral nerve regeneration: In vitro and in vivo studies. J Trauma, 2010; 68: 434-40

27. Yang F, Yan G, Li Y et al: Astragalus polysaccharide attenuated iron overload-induced dysfunction of mesenchymal stem cells via suppressing mitochondrial ROS. Cell Physiol Biochem, 2016; 39: 1369-79

28. Li XT, Zhang YK, Kuang HX et al: Mitochondrial protection and anti-aging activity of Astragalus polysaccharides and their potential mechanism. Int Mol Sci, 2012; 13: 1747-61

29. Rui DP, Bortolanza M, de Castro Issy AC et al: Dopaminergic neurons in Parkinson's disease. Handbook of Neurotoxicity, 2014; 753-88

30. Bubak AN, Redmond DE, Elsworth JD et al: A potential compensatory role for endogenous striatal tyrosine hydroxylase-positive neurons in a nonhuman primate model of Parkinson's disease. Cell Transplant, 2015; 24: 673-80

31. Wang $\mathrm{H}$, Liang $\mathrm{X}$, Wang $\mathrm{X}$ et al: Electro-acupuncture stimulation improves spontaneous locomotor hyperactivity in MPTP intoxicated mice. PLoS One, 2013; 8: e64403

32. Liu Y, Song Y, Zhu X: MicroRNA-181a regulates apoptosis and autophagy process in Parkinson's disease by inhibiting p38 mitogen-activated protein kinase (MAPK)/c-Jun N-terminal kinases (JNK) signaling pathways. Med Sci Monit, 2017; 23: 1597-606

33. Nunnari J, Suomalainen A: Mitochondria: In sickness and in health. Cell, 2012; 148: 1145-59

34. Ya L, Lu Z: Differences inABCA1 R219Kpolymorphisms and serum indexes in Alzheimer and Parkinson diseases in Northern China. Med Sci Monit, 2017; 23: 4591-600

35. Lin MT, Beal MF: Mitochondrial dysfunction and oxidative stress in neurodegenerative diseases. Nature, 2006; 443: 787-95

36. Yi R, Min Y, Chen S et al: CD200 inhibits inflammatory response by promoting KATP channel opening in microglia cells in Parkinson's disease. Med Sci Monit, 2016; 22: 1733-41

37. Danielson SR, Held JM, Schilling B et al: Preferentially increased nitration of alpha-synuclein at tyrosine-39 in a cellular oxidative model of Parkinson's disease. Anal Chem, 2009; 81: 7823-28

38. Barnwal B, Karlberg H, Mirazimi A et al: Non-structural protein of CrimeanCongo hemorrhagic fever virus disrupts mitochondrial membrane potential and induces apoptosis. J Biol Chem, 2016; 291: 582-92

39. Cheng EHYA, Wei MC, Weiler $\mathrm{S}$ et al: BCL-2, BCL-XL sequester BH3 domainonly molecules preventing BAX- and BAK-mediated mitochondrial apoptosis. Mol Cell, 2001; 8: 705-11

40. Teijido O, Dejean L: Upregulation of $\mathrm{Bcl} 2$ inhibits apoptosis-driven BAX in sertion but favors BAX relocalization in mitochondria. FEBS Lett, 2010; 584 3305-10 